\title{
Frequency-Domain Analysis of the Periodically-Forced Josephson-Junction Circuit
}

\author{
Sergio Sancho and Almudena Suarez
}

\begin{abstract}
In this paper, a new frequency domain technique to analyze the Josephson-junction circuit dynamics is presented. This technique overcomes some of the limitations inherent to the analytical and time-integration techniques used in previous works. The technique can be extended to the analysis of this device when combined with distributed elements in microwave systems. It allows an efficient analysis of the different types of steady-state solutions and the bifurcation loci in the presence of a periodic driving current source. No restriction is imposed to the driving source amplitude, enabling an accurate analysis of the influence of this parameter on the device superconducting properties. The technique has also been applied to analyze the quasi-periodic states present in this device together with the synchronized solutions to the driving current source.
\end{abstract}

Index Terms-Josephson-junction circuit, nonlinear dynamics, CAD, frequency domain techniques

\section{INTRODUCTION}

$\mathbf{T}$ HE Josephson junction circuit model with ac forcing is used in several applications, such as the superconducting quantum interference devices (SQUID) [1]-[3]. The dynamics of this circuit with small forcing has been analyzed in [3][12] with analytical approaches and time-domain techniques. These techniques predict the junction behavior when driven by a current source of small amplitude, and the analyses are carried out in terms of the system steady-state solutions. In those papers, the range of existence of the superconducting states and nonlinear phenomena such as the hysteresis and the synchronization of the system autonomous solution to the driving current source have been studied.

In those previous works, the used analytical and timedomain techniques present certain limitations for the efficient analysis of the bifurcation loci that delimit the superconducting region and the synchronized ranges. On one hand, some analytical techniques are valid for small value of the driving generator amplitude. On the other hand, when different steady-state solutions coexist in the phase space, the time domain integration presents difficulties to converge to solutions with reduced stable manifolds. In those cases, the time integration can converge to other solutions that are undesired for the performing analysis.

In the case of periodic solutions of lumped circuits, these problems can be overcome by using the time domain

Authors are with the Department of Communications Engineering, ETSIIT, University of Cantabria, Santander, 39005, Spain. Ph: (34)942203912, Fax: (34)942201488 e-mail: sanchosm@unican.es, suareza@unican.es. This work was supported by the Spanish Ministry of Economy and Competitiveness under Contract TEC2011-29264-C03-01.

Copyright (c) 2013 IEEE. Personal use of this material is permitted. However, permission to use this material for any other purposes must be obtained from the IEEE by sending an email to pubs-permissions@ieee.org. continuation techniques described in [13]-[18]. These methods are very powerful, and are applicable to a high number of nonlinear systems. Some of these techniques are based on finite differences, discretizing the state variables along the time interval using a one-dimensional mesh of samples, which are the unknowns to be solved. Other techniques, like shooting, reduce the number of unknowns to a set of initial conditions from which a system ODE is integrated over one period [15], [18]. In the case of quasi-periodic solutions, time-continuation techniques like [16], [17] convert the initial ODE in a system of PDEs. To solve this system, the torus solution is discretized in a n-dimensional mesh, each dimension corresponding to a phase variable. The full-discretization method in [17] makes use of n-dimensional Fourier polynomials in a similar fashion as the technique presented in this paper.

The mentioned simulation problems also arise in the simulation of phase-locked loops (PLL) whose dynamics, in certain topologies, is similar to the Josephson junction circuit [19]. This similarity suggests that the analysis of the Josephson junction circuit in the frequency domain could overcome some of these limitations, in the same way as it has been useful for PLLs in [20], [21]. In this paper, a new frequency-domain technique to analyze the junction dynamics is presented. This technique makes use of the harmonic balance method to express the system equations. It can be extended to simulate this device when embedded in microwave circuits and systems. Some examples of these systems are the combination of Josephson junctions with transmission lines [22], [23], their application to produce microwave mixers [24], [25] and RF nanoelectronic devices, such as oscillators, detectors and frequency converters [26]. These systems are usually simulated in the frequency domain due to several reasons. On one hand, in this domain the linear distributed components with arbitrary frequency responses can be easily, yet quickly, modeled. Lumped element approximations are no longer required and time domain convolution is replaced with simple frequency domain multiplication. Simulating such elements in the time domain by means of convolution can result in problems related to accuracy, causality, or stability. This is especially important for RF, microwave and millimeter frequencies, which are often characterized with measured frequency data. Linear component measures are also made using phasors by network analyzers and are compatible with harmonic balance [18], [27], [28].

By using the frequency domain technique the circuit steady states can be accurately approached by a very small number of harmonic components. As stated in [18], when the circuit contains signals representable by Fourier series with few 
terms, the harmonic balance deals with a smaller number of unknowns than finite differences. This is more evidenced in the case of quasi-periodic solutions, where finite differences techniques use a two-dimensional mesh of samples. On the other hand, this economy in the stored amount of data for each solution is also achieved by the shooting techniques [15]. Then, when compared with the shooting techniques, the frequency domain analysis does not represent an improvement in terms of economy of data.

The frequency domain analysis allows to analyze the whole space of stable and unstable steady-state solutions, providing a global understanding of the circuit dynamics in terms of the system parameters. In contrast to the previous analyses, this technique does not impose any restriction to the driving source amplitude. This will allow to perform a complete analysis of the influence of this amplitude on the superconducting region of the space of parameters. The harmonic balance method is enhanced by using additional equations to directly converge to the bifurcation locus that delimits the super-conducting region. The presented technique allows the efficient simulation of the hysteresis ranges and the boundaries between different ranges of behavior. The frequency domain formulation also enables an efficient stability analysis of the obtained solutions.

For certain values of the circuit parameters, the circuit presents an autonomous oscillating solution which, when mixing with the driving source signal, becomes quasi-periodic. The oscillating solution can also get synchronized to the driving current source [4], [5], [7]. In that case, the resulting synchronization ranges give rise to flat regions in the circuit I-V characteristic [5], [6], [8]. Here, the presented frequency domain technique has been modified using a special set of state variables to obtain the quasi-periodic regimes and the synchronization ranges through a simple parametric analysis. This analysis avoids the convergence to the coexisting superconducting states. The use of additional constraints assures convergence to the two-torus solutions and, in the case of synchronized states, enables the direct calculation of the socalled Arnold tongues.

The paper is organized as follows: in Section II, the new technique is applied to analyze the ranges of existence of the junction superconducting states. In Section III, the quasi-periodic states and the hysteresis phenomenon are analyzed. In this Section, a modification of the technique using a special set of state variables to efficiently simulate the synchronization regimes is also presented. Along the paper, the simulation results have been compared with time domain techniques, obtaining a similar accuracy. In the case of periodic solutions, the results have been validated with time continuation techniques by making use of the toolbox AUTO [13].

\section{ANALYSIS OF THE SUPERCONDUCTING STATES}

The Josephson junction driven by a current source can be modeled by the circuit of Fig. 1, which is described by the following nonlinear equation [8], [11], [12] :

$$
i_{s}(t)=I_{0} \sin \phi+\frac{\hbar}{2 e R} \dot{\phi}+\frac{\hbar C}{2 e} \ddot{\phi}
$$

where $\hbar$ is Planck's constant (divided by $2 \pi$ ), $e$ the electronic charge, $I_{0}$ a threshold current associated with the tunneling current, $C$ the junction capacitance, $R$ the junction resistance and $i_{s}(t)$ the driving source. In this paper, the case of a periodic current source $i_{s}(t)=I_{d c}+I_{a c} \sin \nu t$ will be analyzed. In the quantum mechanical model of the Josephson junction the quantity $\phi$ is the phase difference of the condensate wave functions inside each of the two electrodes that form the junction [3], [12]. This phase is related to the voltage across the junction by the fundamental law:

$$
v(t)=\frac{\hbar}{2 e} \dot{\phi}(t)
$$

Following the normalization provided in [8], equation (1) can be represented by the following two-dimensional system of nonlinear differential equations:

$$
\dot{\phi}=y, \dot{y}=\frac{1}{\beta}(-y-\sin \phi+\rho+A \sin \omega t)=f(\phi, \dot{\phi}, t)
$$

where $\beta=2 e I_{0} C R^{2} / \hbar, \rho=I_{d c} / I_{0}, A=I_{a c} / I_{0}$, the time variable $t$ has been properly normalized as in [8] and $\omega=\nu \hbar / 2 e R I_{0}$ is the normalized frequency of the driving source. Due to the periodicity of the system (3) on the variable $\phi$, we can represent the state variables $(\phi, y)$ in the phase space $S^{1} \times \Re$, with $S^{1}=[-\pi, \pi]$. It is considered that the junction behaves as a superconductor when a non-zero average current can be maintained through the device with zero average voltage across it. In the presence of the driving source, the average current flowing through the junction is $\left\langle i_{s}(t)\right\rangle=I_{d c}$, with \langle\rangle being the time average over the driving source period. Then, the supercurrent is obtained when $I_{d c} \neq 0$ and at the same time $\langle v(t)\rangle=0$. In the absence of periodic forcing $(A=$ 0 ), for $|\rho|<1$ the system (3) has in the phase space a pair of equilibrium points $\left(\phi=\phi_{s}, y=0\right)$ and $\left(\phi=\phi_{n}=\pi-\right.$ $\left.\phi_{s}, y=0\right)$ of saddle and node type, respectively. For $\rho \neq 0$, in these states the junction behaves as a superconductor, since for an applied average current $I_{d c}=\rho I_{0}$ it presents a voltage $v=y \hbar / 2 e=0$. When varying $\rho$, the maximum supercurrent value is $I_{d c}= \pm I_{0}$, attained at $\rho= \pm 1$, where both states collide in the phase space in a saddle-node bifurcation [5], [8].

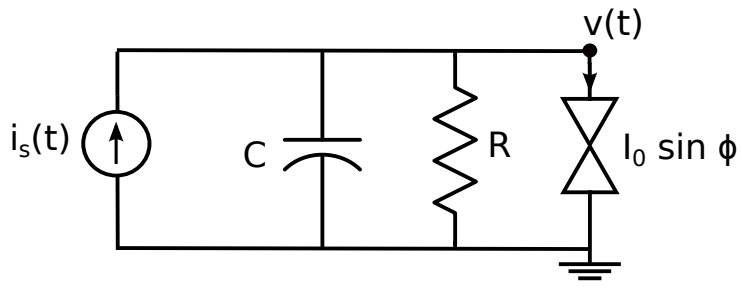

Fig. 1. Schematic of the Josephson junction circuit model.

When a small periodic forcing is present, the equilibrium points become a pair of small amplitude periodic cycles of node and saddle type [6], [8], [29]. In order to analyze these solutions in the frequency domain, they will be expressed in a Fourier series as:

$$
\phi(t)=\sum_{k=-N}^{N} \Phi_{k} e^{j k \omega t}, \quad y(t)=\sum_{k=-N}^{N} j k \omega \Phi_{k} e^{j k \omega t}
$$


where $N$ is the number of harmonics considered. When the system converges to the stable node solution, by introducing the expressions (4) in equation (2), it is obtained that the voltage average value is $\langle v\rangle=\langle y\rangle \hbar / 2 e=0$. The circuit in this case behaves as a superconductor since, for $\langle v\rangle=0$, the average current across the junction is $\left\langle i_{s}(t)\right\rangle=I_{d c}=\rho I_{0}$. The region of the parameters space $(\rho, \beta, A, \omega)$ in which the stable node exists, and therefore superconductivity can be observed, will be denoted in the following by SC region. If now we introduce the expressions (4) into the system (3) and equate the harmonic components at the same frequencies, we obtain the following Harmonic Balance (HB) system:

$$
\bar{H}=[j \bar{k} \omega]^{2} \bar{\Phi}-\bar{F}[\bar{\Phi}]=\overline{0}
$$

where $\bar{\Phi}$ and $\bar{F}$ are the vectors containing the $2 N+$ 1 harmonic components of the time domain signals $\phi(t)$ and $f(t)$. The diagonal matrix $[j \bar{k} \omega]$ multiplies each $k$-th harmonic component in $\bar{\Phi}$ by $j k \omega$. The nonlinear algebraic system (5) can be numerically solved by using the NewtonRaphson technique for a given value of the system parameters.

Our first purpose is to analyze the evolution of the SC region as the forcing amplitude $A$ is increased. In order to simulate the periodic superconducting solution, $N=2$ harmonics have been used. With the aim to allow convergence, we have started the analysis from the value $A=0$, for which the solutions (4) reduce to the equilibrium points $\left(\phi_{n}, \phi_{s}\right)$. Then, we set $\phi_{n}$ as initial condition to (5) and vary $A$ to obtain the solution path. Note that formulation (5) does not impose any restrictions on the magnitude of the amplitude $A$ to obtain the solution paths. The results are shown in Fig. 2, where the harmonic component $\Phi_{0}$ has been represented versus the forcing amplitude $A$. As can be seen, as the normalized source amplitude is increased both periodic solutions get closer in the phase space and finally collide in a turning point. Several values of the parameter $\rho$ have been considered. For small $|\rho|$ value, the resulting paths present a wide region of very high slope around the turning point in the $\Phi_{0}$ variable. This makes it necessary the use of a continuation technique based on the interchange of parameters [30]. In these cases, the error function depends on both the phase harmonics and the forcing amplitude. Then the equation $\bar{H}[\bar{\Phi}, A]=\overline{0}$ is solved at each point of the path by fixing $A$ or $\Phi_{0}$, depending on the magnitude of the derivative $D=\partial \Phi_{0} / \partial A$ at that point. For small $D$ values, $P=\bar{\Phi}$ is the set of unknowns and the parameter $A$ is fixed during the resolution. Near the turning point, for high $D$ values, the variable $\Phi_{0}$ is substituted in $P$ by the parameter $A$ and $\Phi_{0}$ remains fixed during the resolution.

In order to validate the technique, the periodic solutions of each path have been calculated through the time discretization technique implemented in AUTO [13]. The number of mesh intervals used for discretization is NTST $=15$. For each solution, the average value $\Phi_{0}=\langle\phi(t)\rangle$ has been superimposed in Fig. 2. From this comparison, it is derived that the presented frequency domain technique is able to simulate the periodic regimes with the same accuracy as the time continuation technique. On one hand, system (5) contains a single state variable, described with $N=2$ harmonic components. This means $u=2 N+1=5$ real unknowns to be solved by Newton-Raphson's method. We think that, in this particular system, the ability to describe the superconducting region by using $u=5$ real numbers for each solution can be an advantage with respect to the time-domain techniques based on finite differences. These techniques discretize the state variables along the time interval using a one-dimensional mesh of more than $u=5$ components. On the other hand, the time continuation technique is faster than the implemented frequency domain continuation. In the same machine, Intel Core(TM)2 Duo CPU E8500 @ 3.16 GHz, the total simulation time to obtain one path is $T_{s}=1.53 \mathrm{sec}$ for the frequencydomain technique and $T_{s}=0.37 \mathrm{sec}$ for the time continuation.

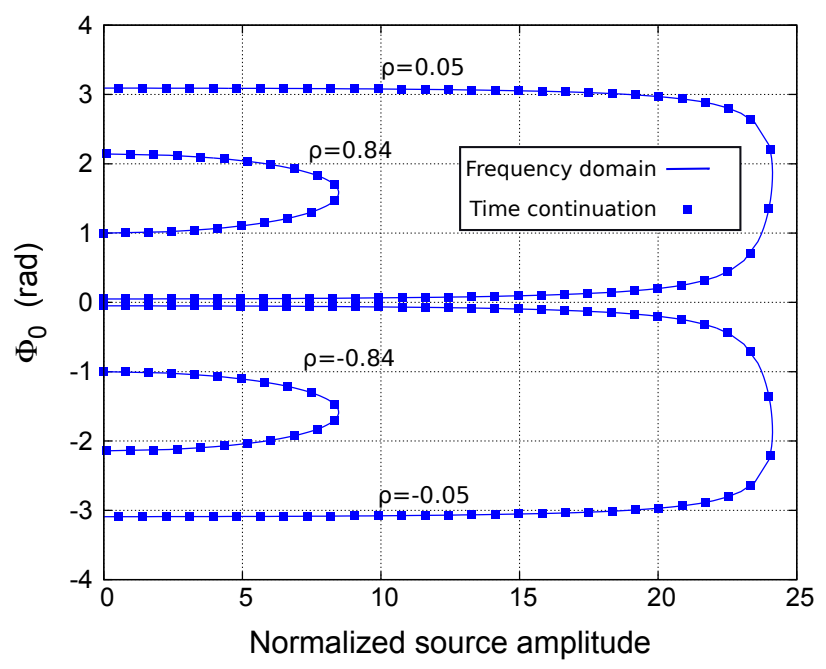

Fig. 2. Influence of the forcing amplitude $A$ over the periodic solutions for $\beta=0.05, \omega=3 \pi \mathrm{rad} / \mathrm{s}$. Paths in the $\left(A, \Phi_{0}\right)$ space. As the parameter $A$ increases, the periodic solutions get closer in the phase space and eventually collide in a turning point. For small $|\rho|$ value the use of an interchange of parameters technique has been necessary. The results of the time domain simulations of the stable solutions have been superimposed for comparison.

Now, with the aim to analyze the global dependence of the $\mathrm{SC}$ region on the $\mathrm{AC}$ normalized amplitude $A$, we have represented the solution paths obtained through the previous technique in the space $\left(A, \rho, \Phi_{0}\right)$. The resulting solution paths can be seen in Fig. 3(a). This representation shows that for $A=0$ the equilibrium points lie in the curve $\sin \phi=\rho$, with $\phi \in S^{1}$. In this case, the maximum supercurrent value is $I= \pm I_{0}$. As the parameter $A$ is increased, the solution paths lie on a surface $S$ in this space, bounded in the $A$ direction by turning points. The SC region for a given value $A_{0}$ of the forcing amplitude is given by the intersection of the surface $S$ in the parameter space $\left(A, \rho, \Phi_{0}\right)$ with the plane $A=A_{0}$. The evolution of the SC region as the amplitude $A$ is varied has been analyzed with the aid of system (5). For each value $A=A_{0}, \rho$ is added to the set of unknowns and $\Phi_{0}$ is considered as a parameter. In this way, as can be seen in Fig 3(a), for each value of $\Phi_{0}$ there exists a unique solution for the parameter $\rho$. Solving system (5) for a sweeping of $\Phi_{0}$ in the interval $[-\pi, \pi]$, a path in the plane $\left(\rho, \Phi_{0}\right)$ is obtained. The resulting paths are shown in Fig. 3(b). In each path a pair of periodic solutions coexist which, as will be shown in the next subsection, are of node and saddle type, respectively. For each value of the amplitude $A$ the SC region is limited by a pair of 
turning points at $\rho= \pm \rho_{s}$. In this case, the technique has also been validated through the time domain integration of system (3). As in the previous case, the transient states become longer for $\rho$ values in the vicinity of the turning points, increasing the simulation computational cost. The results are superimposed in Fig. 3(b).

As can be seen, as $A$ is increased the interval $\left[-\rho_{s}, \rho_{s}\right]$ gets reduced in size. Therefore, the maximal admissible supercurrent $I_{d c}= \pm \rho_{s} I_{0}$ also decreases with $A$. The maximum supercurrent is attained at the turning points of the paths represented in Fig. 3(b). Since there does not exist an open interval of solutions to system $\bar{H}[\bar{\Phi}, \rho]=\overline{0}$ about these points, the implicit function theorem asserts that:

$$
\operatorname{det}\left[\frac{\partial \bar{H}\left[\overline{\Phi_{t}}, \rho_{s}\right]}{\partial \bar{\Phi}}\right]=0
$$

where $\bar{\Phi}_{t}$ is the value of the set of harmonics at the turning point $\rho=\rho_{s}$. The dependence of the maximum supercurrent value $I_{d c}$ on the input source amplitude $A$ can be obtained efficiently and with high precision by solving system (5)-(6) with the set of unknowns $P=(\bar{\Phi}, \rho)$. The results are shown in Fig. 4. In this figure, the normalized maximum supercurrent $\left|I_{d c}\right| / I_{0}=\left|\rho_{s}\right|$ has been represented versus the amplitude $A$, for $\beta=0.05$ and $\omega=3 \pi \mathrm{rad} / \mathrm{s}$. For these values, the SC region exists in the range $A \in[0,25]$.

\section{A. Stability analysis}

In order to analyze the stability of the periodic solutions of system (3), we study the trajectory of the state variables in the neighborhood of the cycles. In this situation, the state variables can be expressed as:

$$
\begin{aligned}
& \phi(t)=\sum_{k=-N}^{N}\left[\Phi_{k}+\Delta \Phi_{k}(t)\right] e^{j k \omega t}, \\
& y(t)=\sum_{k=-N}^{N}\left[j k \omega \Phi_{k}+\Delta Y_{k}(t)\right] e^{j k \omega t}
\end{aligned}
$$

Now, introducing this expression into the system (3), equating the harmonic components at the same frequencies and eliminating the steady-state components, we obtain the following linear system:

$$
\begin{aligned}
& {[j \bar{k} \omega] \Delta \bar{\Phi}+\Delta \dot{\bar{\Phi}}=\Delta \bar{Y},} \\
& {[j \bar{k} \omega] \Delta \bar{Y}+\Delta \dot{\bar{Y}}=T_{G_{\phi}}[\bar{\Phi}] \Delta \bar{\Phi}+T_{G_{y}}[\bar{\Phi}] \Delta \bar{Y}}
\end{aligned}
$$

where $T_{G_{\phi}}[\bar{\Phi}]$ and $T_{G_{y}}[\bar{\Phi}]$ are the Toeplitz matrixes corresponding the harmonic components of the time-domain functions $g_{\phi}(t)=\partial f / \partial \phi$ and $g_{y}=\partial f / \partial \dot{\phi}$ evaluated at the periodic steady state. The equations ( 8 ) constitute a linear time invariant (LTI) system in the perturbation harmonic components, which can be expressed in compact form as $\dot{\bar{X}}=M \bar{X}$ with [31] :

$$
\bar{X}=\left(\begin{array}{c}
\Delta \bar{\Phi} \\
\Delta \bar{Y}
\end{array}\right), M=\left(\begin{array}{cc}
-[j \bar{k} \omega] & I \\
T_{G_{\phi}}[\bar{\Phi}] & T_{G_{y}}[\bar{\Phi}]-[j \bar{k} \omega]
\end{array}\right)
$$

where $I$ is the identity matrix. The stability of the periodic solutions is given by the eigenvalues of the matrix $M$, which

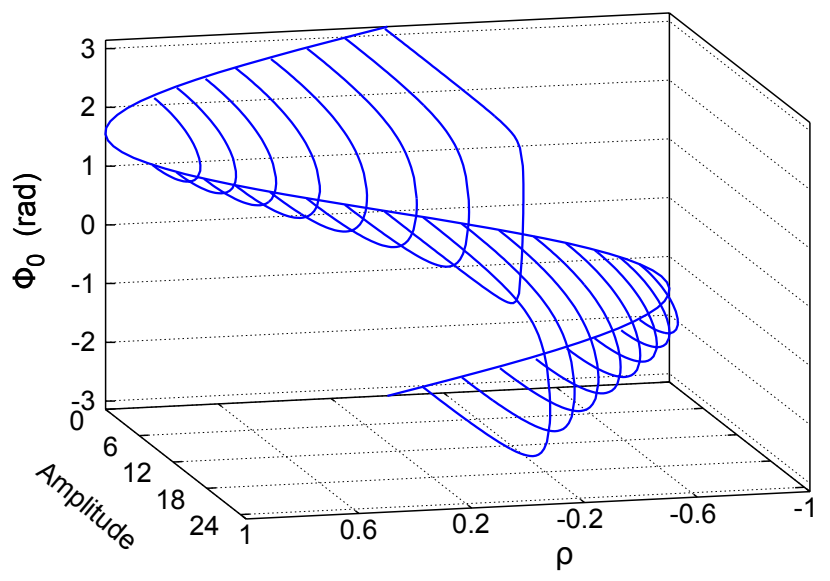

(a)

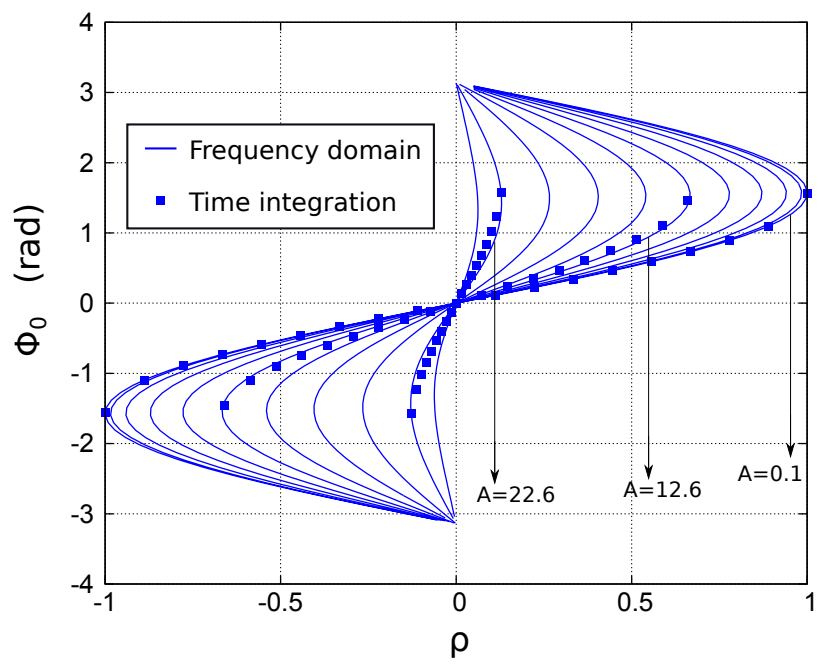

(b)

Fig. 3. Influence of the forcing amplitude $A$ over the periodic solutions for $\beta=0.05, \omega=3 \pi \mathrm{rad} / \mathrm{s}$. (a) Paths in the $\left(A, \rho, \Phi_{0}\right)$ space. For $A=0$ the equilibrium points lie in the curve $\sin \phi=\rho$. The solution paths lie on a surface bounded in the $A$-direction by turning-point bifurcations (b) Evolution of the SC region with the forcing amplitude. The stable solutions obtained through time domain simulations have been superimposed.

here will be called system poles. Taking into account the Toeplitz structure of the matrixes forming $M$, the system poles are given by a set $\left\{\lambda_{i}^{q}\right\}$ that can be expressed as [32], [33] :

$$
\lambda_{i}^{q}=\sigma_{i}+j\left(\omega_{i}+q \omega\right), \quad i=1,2, \quad q=-N, \cdots, N
$$

These poles can be mapped to a pair of Floquet multipliers $m_{i}=e^{2 \pi \lambda_{i}^{q} / \omega}$ for all $q$ and $i=1,2$. The Floquet multipliers have been evaluated along the paths of Fig. 2, with the result of Fig. 5. Since the stability properties of these paths are symmetrical about $\rho=0$, only the analyses corresponding to positive values of $\rho$ have been shown. In this figure, the magnitude of $m_{1}$ has been traced, after ordering the multipliers to fulfill $\left|m_{1}\right|>\left|m_{2}\right|$. The module $\left|m_{2}\right|$ remains always smaller that the unity, so it has not been traced in the figure. As can be seen, the two periodic solutions that coexists for each $\rho$ value are of saddle and node type along the whole range of the amplitude $A$ covered in each path. At the turning points the magnitude of $m_{1}$ crosses the value $\left|m_{1}\right|=1$ with infinite slope, which corresponds to a saddle-node bifurcation. 


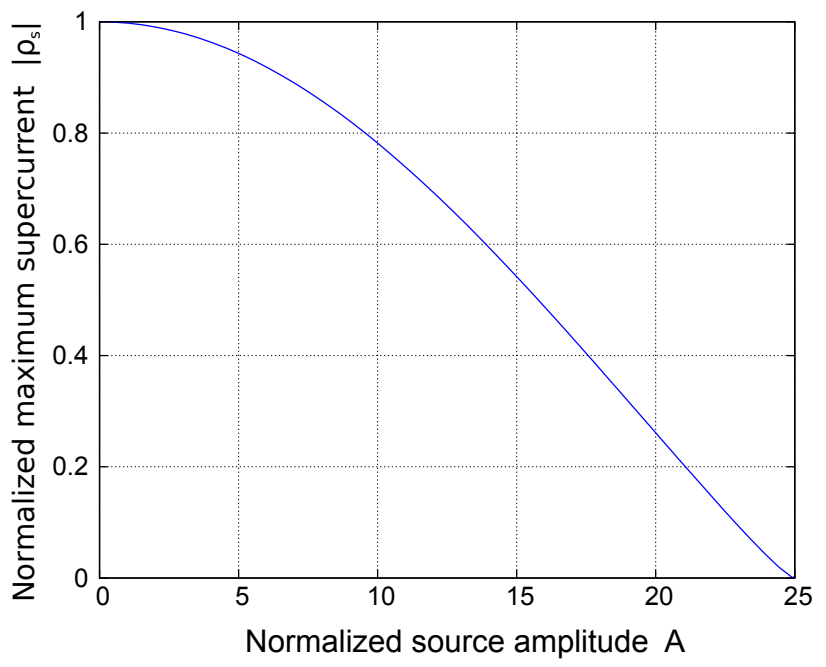

Fig. 4. Dependence of the maximum supercurrent on the input source amplitude $A$ for $\beta=0.05$ and $\omega=3 \pi \mathrm{rad} / \mathrm{s}$

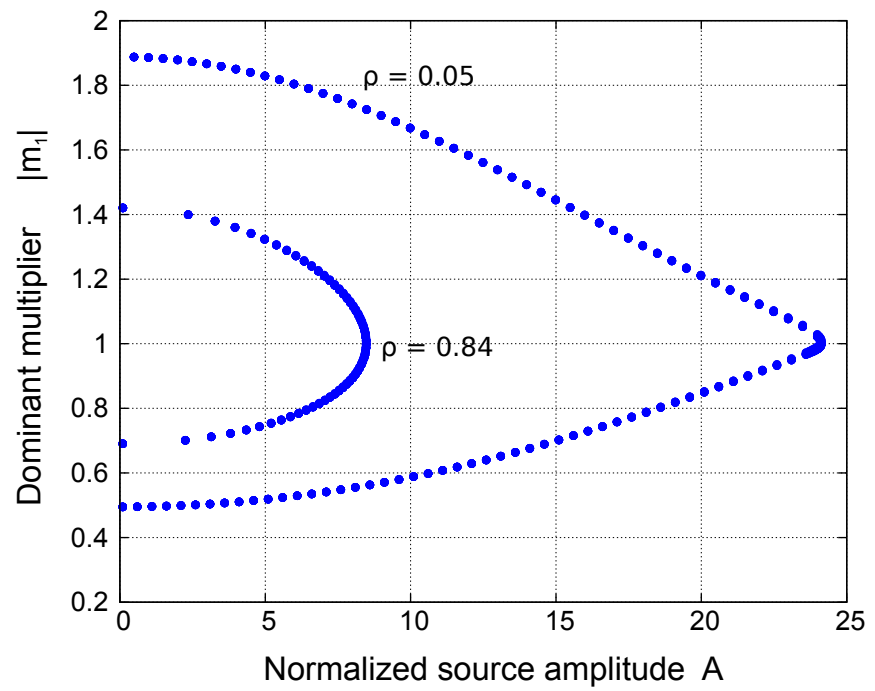

Fig. 5. Stability analysis of the periodic solutions in terms of the forcing amplitude $A$. The periodic solutions are of saddle and node type and the turning point observed in the analysis of Fig. 2 corresponds to a saddle-node bifurcation

\section{ANALYSIS OF THE TWO-TORUS SOLUTION}

\section{A. Steady-state solution}

As stated in [5], [6], [8], for $0<|A|<\epsilon$ with $\epsilon$ small, as the parameter $\rho$ is increased from $\rho=0$ there exists a stable solution which lies on a two dimensional stable (attracting) invariant surface in the state space $(\phi, \dot{\phi}, \varphi=\omega t)$ [8]. This surface is periodic in $\phi$ and $\varphi$ and so is diffeomorphic to a twotorus. In this solution, the state variable $\phi$ has the following form:

$$
\phi(t)=\omega_{a} t+\theta(t)
$$

where $\theta(t)$ is a bounded signal containing the frequencies $\omega$ and $\omega_{a}$. The new frequency $\omega_{a}$ is autonomous, depending on the system parameters. The phase variable $\phi(t)$ in (11) grows unbounded with time when considered as an element of $\Re$. This makes it difficult to approach it by a finite Fourier basis in an Harmonic Balance system of the form (5). In order to overcome this problem, we perform the change of variables $(\phi, y) \rightarrow\left(\theta=\phi-\omega_{a} t, y\right)$, so that the system (3) becomes:

$$
\begin{aligned}
& \dot{\theta}=y-\omega_{a}, \quad \dot{y}=u(\theta, \dot{\theta}, t), \\
& u(\theta, \dot{\theta}, t) \equiv \frac{1}{\beta}\left[-y-\sin \left(\omega_{a} t+\theta\right)+\rho+A \sin \omega t\right]
\end{aligned}
$$

Note that, when expressed in the variables $(\theta, y)$, the twotorus solution becomes bounded in the space $\Re \times \Re$. System (12) could be simulated using time continuation techniques like [16], which converts (12) in a system of PDEs. To solve this system, the torus solution is discretized in a ndimensional mesh, each dimension corresponding to a phase variable. The number of samples of each phase variable must be high enough to properly construct the phase derivatives of the system of PDEs. As stated in [16], for $n=2$ the twodimensional mesh can be composed of several hundreds of samples. This technique is very powerful, and is applicable to a high number of nonlinear systems. In other works like [17], two techniques are presented: the semi-discretization method and the full-discretization method. In the semi-discretization technique, the state variables are expressed as Fourier series with time-varying coefficients. The fundamental frequency of the series is given by the forcing term, whereas the timevarying coefficients are periodic with the system autonomous frequency. These expressions are introduced in the original system of EDOs and, applying the Galerkin's method, the equations equating harmonics of the same frequencies are obtained. As a result, the original system is transformed into an autonomous system of EDOs whose solution is periodic. In [17], this final system is solved by time-continuation techniques. In the full-discretization method, the state variables are expressed in multi-dimensional Fourier series with constant coefficients. The dimension of the series is given by the number of independent frequencies contained in the solution. These expressions are introduced in the original system of EDOs and the result is a discretized system to be solved. In the particular case of the Josephson junction circuit, we have used a similar frequency- domain description of the state variables as in the full-discretization method. One reason is the reduced number of harmonic components describing the quasi-periodic solution in the frequency domain. Other reasons for using a frequency-domain approach have been given in the Introduction. The Fourier expression of the state variables corresponding to this solution is:

$$
\begin{aligned}
& \theta(t)=\sum_{k, l} \Theta_{k, l} e^{j\left(k \omega t+l \omega_{a}\right) t}=\sum_{r} \Theta_{r} e^{j \Omega_{r} t}, \\
& y=\sum_{r} Y_{r} e^{j \Omega_{r} t}, k=-N, \ldots, N, \quad l=-M, \ldots, M
\end{aligned}
$$

with $\bar{\Omega}=\left[\Omega_{1}, \ldots, \Omega_{(2 N+1)(2 M+1)}\right]^{t}$ being the vector frequency basis containing all the frequency components $k \omega+l \omega_{a}$. Now, introducing the expression (13) in the system (12) we arrive to the Harmonic Balance system:

$$
\begin{aligned}
& \bar{H}\left[\bar{\Theta}, \omega_{a}\right]=[j \bar{\Omega}] \bar{Y}\left[\bar{\Theta}, \omega_{a}\right]-\bar{U}\left[\bar{\Theta}, \omega_{a}\right]=\overline{0}, \\
& \bar{Y}\left[\bar{\Theta}, \omega_{a}\right] \equiv[j \bar{\Omega}] \bar{\Theta}+\omega_{a} \overline{1}_{d c}
\end{aligned}
$$

where $\bar{\Theta}, \bar{Y}$ and $\bar{U}$ are the vectors containing the $(2 N+$ $1)(2 M+1)$ harmonic components of the time domain signals 
$\theta(t), y(t)$ and $u(t)$. The diagonal matrix $[j \bar{\Omega}]$ multiplies each $k$-th harmonic component by $j \Omega_{k}$ and the vector $\overline{1}_{d c}$ provides the harmonic components of the signal $x(t)=1$ in the basis $\bar{\Omega}$. The unknowns of system (14) are the harmonic components $\bar{\Theta}$ and the autonomous frequency $\omega_{a}$. In order to assure the convergence to the two-torus solution, the following equations can be added to system (14) :

$$
\frac{\omega_{a}-\lambda}{\lambda}=0, \quad \Theta_{01}^{r}=0
$$

where the superindex $r$ means real part and $\lambda$ is an additional unknown to be solved. The left equation in (15) assures that the obtained solution has the form (11), avoiding convergence to the periodic limit cycle solution (with $\omega_{a}=0$ ). The equation in the right is used to balance system (14)(15), due to the system self-oscillation at the frequency $\omega_{a}$. The introduction of this additional equation is justified in the Appendix A. As in the case of the system (5), the nonlinear algebraic system (14)-(15) can be numerically solved by using the Newton-Raphson technique. First, the case $A=0$ has been analyzed. In this case, the solution that lies on the twotorus is periodic in the space $\left(S^{1}, \Re\right)$ and is called rotation [8]. The autonomous frequency $\omega_{a}$ of this solution has been represented in Fig. 6 as the parameter $\rho$ is swept from outside to inside of the SC region. As described in [8], as the parameter $\rho$ is reduced the rotation solution approaches the $y=0$ axis in the phase space $(\phi, y)$. Inside the region $|\rho| \leq 1$, the rotation coexists in the phase space with the saddle and node equilibrium points corresponding to the superconducting states, described in Section II. In this region, the system will evolve to one or another solution depending on the initial conditions, given rise to the hysteresis phenomenon. The rotation eventually collides with the equilibrium point of saddle type and disappears in a saddle-connection bifurcation [6], [8].

The hysteresis phenomenon can be explained in the following way [8]. Let the system parameters be out of the superconducting (SC) region. Then, a non superconducting state is observed, whose corresponding solution lies in a twotorus in the phase space. Let the system parameter(s) be now moved from this point to another point $\mathrm{H}$ inside the $\mathrm{SC}$ region where the two torus and the limit cycle solutions coexist. We will continue observing the the two-torus solution because the initial conditions are near this kind of solution. Then, we see a non superconducting solution at the point H. Now, we further move the system parameter(s) from $\mathrm{H}$ to a point $\mathrm{S}$ inside the SC region where only the limit cycle solution exists. In $\mathrm{S}$, the system trajectory evolves to the limit cycle and a superconducting state is observed. Now, if the system parameters are returned to the point $\mathrm{H}$ in a reversible way, the state variables are maintained inside the stable manifold of the limit cycle. As a consequence, we observe now a superconducting state in $\mathrm{H}$. Then, the observed state in $\mathrm{H}$ depends on the path followed by the system parameters.

Since the simulation of the frequency domain system (14)(15) assures the convergence to the rotation solution, the hysteresis zone and the evolution of the autonomous frequency $\omega_{a}$ can be easily calculated through this technique. Note that, as explained in [5]-[8], [12] for $A=0$, as the parameter $\beta$ is reduced the hysteresis region shrinks and eventually disappears. In the presented analysis, the value $\beta=5$ providing hysteresis has been chosen to prove the ability of the new technique to converge to the two-torus solution when coexisting with the periodic superconducting solution.

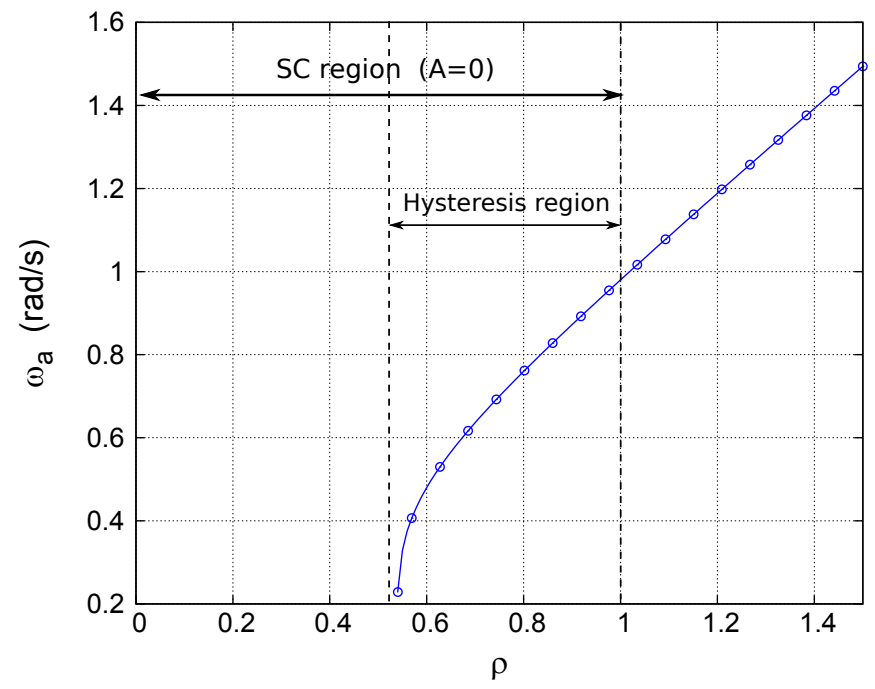

Fig. 6. Evolution of the rotation solution $(A=0)$ with the parameter $\rho$, for $\beta=5$. The autonomous frequency has been represented as a function of $\rho$.

Next, the two-torus solution for $|A|>0$ is analyzed. The vector $\Theta$ is constituted by a set of 12 harmonic components, resulting in a number of $u=25$ unknowns to be solved. In this case, the previous rotation-type solution is in general transformed to a quasi-periodic solution, containing the frequency components $\omega_{a}$ and $\omega$. If the parameter $\rho$ is swept from outside to inside of the SC region as in the previous analysis, the behavior of this solution is qualitatively similar to the case $A=0$. For certain values of the parameter $\rho$ the solution in the two-torus gets synchronized to the driving source, as it will be shown in the Section III.B. In the part of the SC region where the two torus coexists with the saddle and node-type periodic cycles, hysteresis can be observed in a manner similar to what happens when $A=0$. As illustration, the two-torus solution has been calculated in the presence of the periodic forcing, for two values of $\rho$. The phase space representation of the steady-state solutions is shown in Fig. 7. The mean value $\langle y(t)\rangle$ agrees with the autonomous frequency $\omega_{a}$, as expected from the expression (11). The solutions obtained through Harmonic Balance and time domain simulations have been depicted together for comparison. The solution presents one degree of freedom in the phase of the autonomous component $\omega_{a}$. When simulated in the frequency domain, the value of this phase component is determined by the right equation in (15). In the time domain simulation, this value will depend on the initial conditions of the trajectory that evolves to the two-torus. As a consequence, the solutions obtained in the time and frequency domains are usually phase shifted, as can be seen in Fig. 7 for the case $\rho=0.55$. 


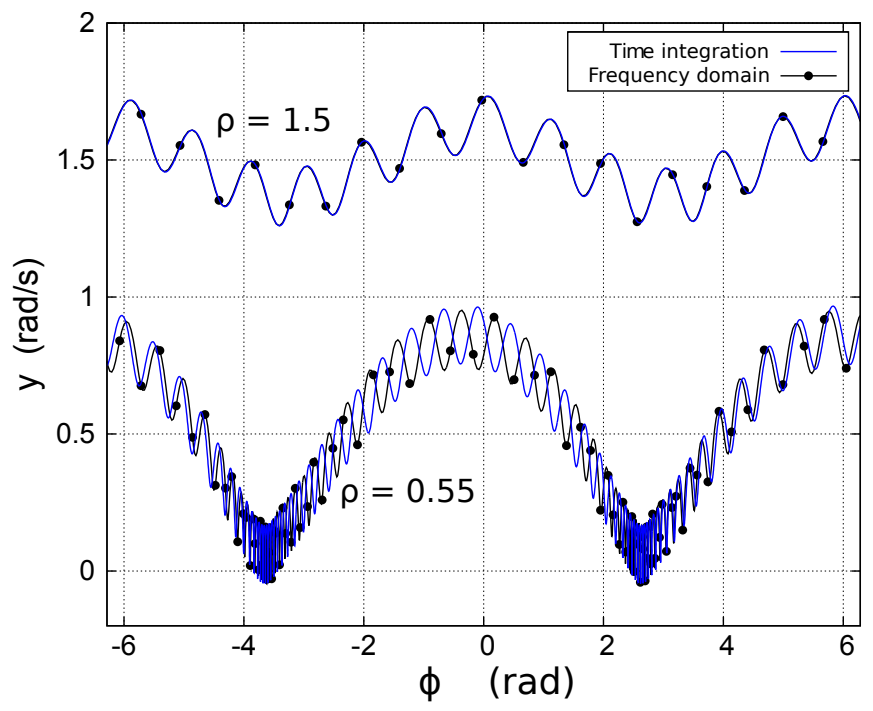

Fig. 7. Two-torus solutions. Phase space representation for $\beta=5$ and $\rho=1.5,0.55$. The periodic forcing parameters are $A=5, \omega=3 \pi \mathrm{rad} / \mathrm{s}$. Comparison with the time-integration simulation

\section{B. Analysis of the synchronized states}

When the frequencies $\omega$ and $\omega_{a}$ are harmonically related in the form $m \omega=n \omega_{a}$ with $m, n \in \mathcal{Z}^{+}$, the solution that lies on the two-torus is periodic. As it has been analyzed in the previous Section, in the absence of the driving current source the autonomous frequency $\omega_{a}$ grows monotonically with the parameter $\rho$. As a result, as the parameter $\rho$ is varied, the autonomous component $n \omega_{a}$ can get synchronized to the $m-t h$ harmonic provided by the source. If the amplitude $A$ is small enough, for a given value of the driving source parameters $(A, \omega)$ the relation $m \omega=n \omega_{a}$ is fulfilled in a closed interval $I(A, \omega, m, n)=\left[\rho_{L}, \rho_{U}\right]$ of the parameter $\rho$, whose length is proportional to the amplitude value $A$ [34], [35].

As can be derived by combining equations (2), (11) and (13), inside each interval $I(A, \omega, m, n)$ the average voltage remains constant at the value $\langle v\rangle=\hbar m \omega / 2 n e$. Then, considering that $I_{d c}=\rho I_{0}$, the synchronized ranges provide flat regions in the $I_{d c}-\langle v\rangle$ representation, as it has been extensively analyzed in [5], [7], [8] through time-domain techniques. With the aim to analyze these ranges in the frequency domain, we can particularize system (12) to the synchronized solutions by making $\omega_{a}=m \omega / n$ :

$$
\begin{aligned}
& \beta \ddot{\theta}+\dot{\theta}+\frac{m \omega}{n}-\rho+f(\theta, t)=A \sin \omega t, \\
& f(\theta, t)=\sin \left(\frac{m \omega}{n} t+\theta\right)
\end{aligned}
$$

In the synchronized state, the variable $\theta(t)$ is periodic and can be expressed in a periodic Fourier series with the fundamental frequency $\omega_{s}=\omega / n$. Then, system (16) can be translated to the frequency domain obtaining the Harmonic Balance system:

$$
\begin{aligned}
\bar{H}[\bar{\Theta}, \rho] \equiv & \left\{\beta\left[j \bar{k} \omega_{s}\right]^{2}+\left[j \bar{k} \omega_{s}\right]\right\} \bar{\Theta}+ \\
& +\overline{1}_{d c}\left(\frac{m \omega}{n}-\rho\right)+\bar{F}[\bar{\Theta}]=A \bar{E}_{n}
\end{aligned}
$$

where $\bar{\Theta}, \bar{F}[\bar{\Theta}]$ and $\bar{E}_{n}$ are the vector of harmonics of the signals $\theta(t), f(t)$ and $\sin \omega t$ in the periodic Fourier basis. The unknowns of system (17) are the $2 N+1$ harmonic components in $\bar{\Theta}$.

Previous analyses like [36] have analyzed the synchronization mechanism of an autonomous oscillation at frequency $\omega_{a}$ by an input source at frequency $\omega \simeq \omega_{a} / m$. In these analyses, it is considered that the nonlinear functions of the system of ODEs are the ones that provide higher harmonic components of the source, in particular the harmonic $m \omega \simeq \omega_{a}$, which synchronizes the autonomous component. Nevertheless, that explanation is no longer valid in the case of the Josephson-junction circuit, since the external input is modeled in the system of ODEs as an additive term, not affected by the nonlinear function $\sin \phi$. The analyses [5], [7] perform a theoretical study of this synchronization phenomenon by using a time-domain description of the system, which require elaborate analyses of the two-torus solution or the use of integral manifolds. The frequency domain description of the system deals directly with the harmonic components involved in the synchronization phenomenon. Then, this formulation allows to derive a simple alternative and analytical explanation for the ultra-synchronization mechanism in these kinds of systems. Other methods to study the synchronized states in periodically-forced circuits and their stability are presented in [37]-[40]. As it is explained in the Appendix B, if the amplitude $A$ is small enough, the set of synchronized solutions of system (17) lie in a closed curve in the space $(\bar{\Theta}, \rho)$. Along this curve, the harmonic component $\Theta_{p}=\left|\Theta_{p}\right| e^{j \varphi_{p}}$, with $p=m n$, remains phase-locked to the $m-t h$ harmonic of the current source. As a consequence, along the synchronization range versus $\rho$, the phase $\varphi_{p}$ of the harmonic component $\Theta_{p}$ covers the range $[0,2 \pi]$. In order to simulate the set of synchronized solutions, the phase $\varphi_{p}$ will be considered as a parameter and it will be varied in the interval $[0,2 \pi]$. To determine the system, the parameter $\rho$ is included in the set of unknowns. Then, the system to be solved is:

$$
\bar{H}[\bar{\Theta}, \rho]=A \bar{E}_{n}, \quad \arg \Theta_{p}=\varphi_{p}
$$

As illustration, system (18) has been solved to obtain the synchronized states for $(m=1, n=3)$. The synchronization range is obtained by sweeping the phase $\varphi_{3}$ in (18) between 0 and $2 \pi$. The result is shown in Fig. 8. Two values of the normalized source amplitude $A$ have been selected, showing that for small $A$ the width of the interval $I(A, \omega, m, n)$ is proportional to this parameter.

Performing an analysis similar to that of Fig. 3(b), it can be seen that these two synchronized-behavior intervals are inside the SC region, coexisting with the periodic cycle solutions corresponding to superconducting states. The system will evolve to one or another solution depending on the initial conditions, giving rise to the hysteresis phenomenon described in [5], [6], [8]. In the synchronized intervals, delimited by a pair of turning points, the $I_{d c}-\langle v\rangle$ characteristic presents flat regions corresponding to ranges of constant average voltage. These regions can be easily obtained with the presented 


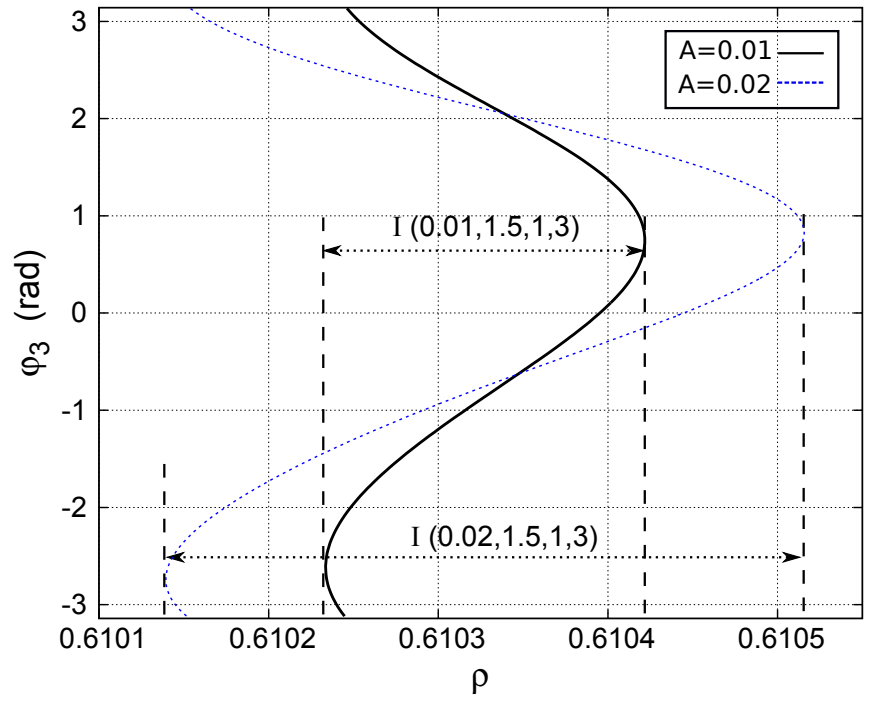

Fig. 8. Harmonic balance simulation of the synchronized states fulfilling $\omega=3 \omega_{a}$ for $\beta=5$ and $\omega=1.5 \mathrm{rad} / \mathrm{s}$. The periodic windows have been obtained for two different values of the normalized amplitude $A$

frequency domain technique, by solving system (18) for the corresponding pairs $(m, n)$.

As can be seen in Fig. 8, for each value of the parameter $\rho$ there exists a pair of synchronized solutions. These solutions are of node and saddle type, colliding at each edge of the synchronization range in a saddle-node bifurcation [34], [35], [41]. The evolution of these bifurcation points with the source amplitude $A$ delimits the location and width of the considered synchronization range for each value of the parameter $A$. This evolution can be analyzed by using the fact that at the turning points the determinant of the HB Jacobian matrix vanishes :

$$
\operatorname{det}\left[\frac{\partial \bar{H}[\bar{\Theta}, \rho]}{\partial \bar{\Theta}}\right]=0
$$

as it corresponds to a saddle-node bifurcation. Then, equation (19) is added to (18) and the resulting system is balanced by introducing $\varphi_{p}$ in the set of unknowns. Fig. 9 shows the result of solving system (18)-(19) versus the amplitude $A$. The same range $(m=1, n=3)$ as in Fig. 8 has been analyzed. In this representation, the synchronized area is delimited by the so-called Arnold tongue [42]. For small source amplitude, the width of the range $I(A, \omega, m, n)$ grows linearly with $A$ but, as the amplitude $A$ is increased, the growing rate becomes nonlinear. There exists a tongue $T(m, n)$ associated to each pair $(m, n)$, and their existence has been theoretically predicted in previous works [5], [7]. The frequency domain technique presented in this Section allows the simulation of these tongues for any pair of values $(m, n)$. In order to verify the technique, a set of time domain simulations of system (3) has been performed to obtain the two torus solution in the range of $\rho$ values of Fig. 9. Note that the time integration simulation of the two-torus in this region can be involved. This is because the stable manifold of the two-torus solution reduces and intertwines with that of the coexisting superconducting solution as $A$ is increased or the limits of the synchronization range are approached. In those cases it is difficult to avoid convergence to the coexisting stable periodic solution. In Fig. 9 the value $A=4$ has been chosen for the time integration simulations. For each simulation, the value of the normalized average voltage $2 e\langle v\rangle / \hbar=\langle\dot{\phi}\rangle$ has been calculated. As can be seen in this figure, the normalized voltage presents a flat region whose limits agree with the $\left(\rho_{L}, \rho_{U}\right)$ values predicted by the frequency domain technique for $A=4$. Inside this region, the normalized average voltage remains constant at the value $2 e\langle v\rangle / \hbar=m \omega / 2 n$.

The technique has also been validated with the toolbox AUTO [13]. The number of mesh intervals used for discretization is NTST $=15$. The detection of saddle-node bifurcations has been activated in the simulation, obtaining the bifurcation locus that delimits the synchronized region. The results are superimposed in Fig 9.

Finally, it must be emphasized that the presented frequencydomain formulation does not impose any restriction to the amplitude of the driving source. According to the synchronization theory [34], [35], as the amplitude of the driving source is increased, the closed synchronization intervals eventually break into open curves. Although this case can be simulated using the presented technique, the analyses have been centered on the case of closed intervals, since no experimental analysis of this phenomenon in the Josephson junction has been found in the literature.

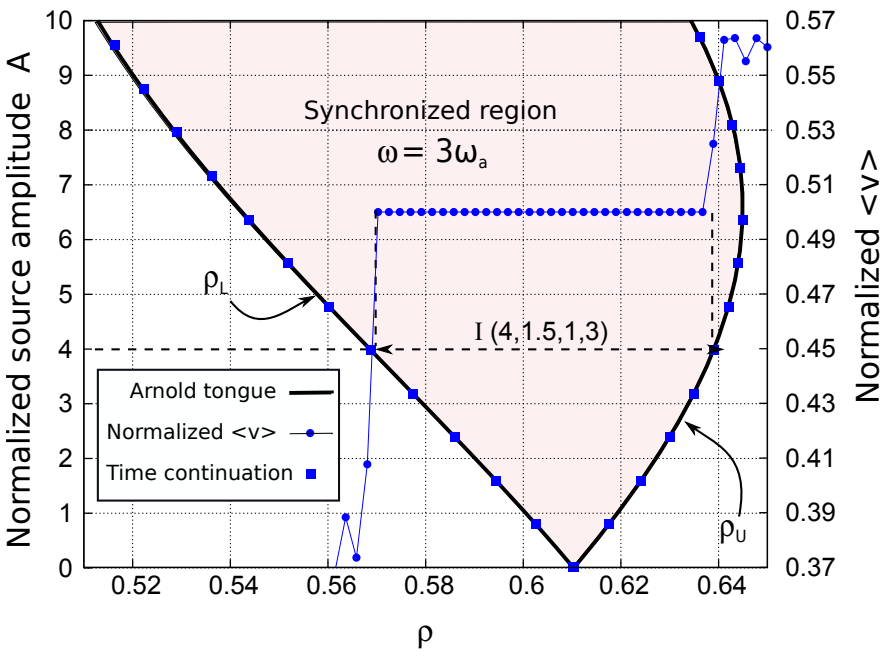

Fig. 9. Evolution of the lower and upper limits of the synchronization range $\omega=3 \omega_{a}$ with the normalized source amplitude $A$ (Arnold tongue), for $\beta=5$ and $\omega=1.5 \mathrm{rad} / \mathrm{s}$. The time integration simulations of the normalized average voltage $2 e\langle v\rangle / \hbar$ for $A=4$, together with the bifurcation locus obtained in AUTO, have been superimposed for comparison

\section{CONCLUSION}

A frequency-domain technique has been proposed to analyze the solutions of the periodically-forced Josephson junction circuit. This technique avoids some of the problems inherent to the time domain integration, such as long transient states or convergence to undesired solutions. It has been used to obtain efficiently and with high precision the bifurcation locus that delimits the SC region in terms of the amplitude A. This analysis has allowed to predict the reduction of the 
maximum supercurrent allowed value as the amplitude $I_{a c}$ of the driving source is increased. The harmonic properties of the synchronized solution have been studied in detail. Making use of these properties, a formulation has been derived to efficiently simulate the quasi-periodic regimes and the width and location of the synchronization ranges that give rise to flat regions in the I-V characteristic. The technique has been validated by comparison with time continuation methods and time integration simulations.

\section{APPENDIX}

A. Analysis of the degree of freedom in the autonomous quasiperiodic solution

Let $\bar{x}_{s}(t)=\sum_{k, l} \bar{X}_{k, l} e^{j\left(k \omega+l \omega_{a}\right) t}$ be a solution to a system of ODEs :

$$
\dot{\bar{x}}=\bar{h}[\bar{x}, t]=\bar{h}[\bar{x}, t+n T], \forall n \in \mathcal{Z}, \quad T=\frac{2 \pi}{\omega}
$$

where $\omega$ and $\omega_{a}$ are not harmonically related. From the periodicity of the function $\bar{h}[\bar{x}, t]$ in the $t$ variable it can be derived that $\bar{x}_{s}(t+n T)$ is a solution for $\forall n \in \mathcal{Z}$. This solution is expressed in the Fourier basis as :

$$
\begin{aligned}
\bar{x}_{s}(t) & =\sum_{k, l} \bar{X}_{k, l} e^{j\left(k \omega+l \omega_{a}\right)(t+n T)}= \\
& =\sum_{k, l} \bar{X}_{k, l} e^{j\left(k \omega+l \omega_{a}\right) t} e^{j l \varphi}, \quad \varphi=2 \pi n \frac{\omega_{a}}{\omega}
\end{aligned}
$$

Now, since $\omega_{a} / \omega$ is an irrational number, by choosing the appropriate value of $n$ we can add to the phase of the $(0, l)$ component any desired phase shift $\varphi$ with any degree of accuracy. In particular, a constraint of the form $\operatorname{Re} \mathrm{X}_{0,1}^{\mathrm{i}}=0$ can be imposed to the solution.

\section{B. Analysis of the synchronized states versus the parameter $\rho$}

In order to simulate the synchronized solution inside the interval $I(A, \omega, m, n)$ we can make use of the following properties :

Proposition 1: Let $\bar{x}(t)=[\theta, y]^{t}$ be the vector of state variables of system (12). Along the interval $I(A, \omega, m, n)$, for $A$ small enough the phase of the $m n-t h$ harmonic of each state variable of the synchronized solution in the basis $\left\{e^{j k \omega_{s} t}\right\}$ covers the range $[0,2 \pi]$.

Proof: As explained in Section III, in the absence of the input current source, the two-torus solution of system (12) becomes periodic. Then, the vector of state variables can be expressed as:

$$
\bar{x}(t)=\sum_{k} \bar{X}_{k} e^{j k \omega_{0} t}, \quad \bar{x}=[\theta, y]^{t}
$$

where $\omega_{0}$ is the value of the autonomous frequency $\omega_{a}$ in the absence of the external source. When this source is introduced, the two-torus solution can be expressed in the following general way:

$$
\bar{x}(t)=\sum_{k, l}\left[\bar{X}_{k, l}+\Delta \bar{X}_{k, l}(t)\right] e^{j\left[k\left(\omega_{0} t+\varphi(t)\right)+l \omega t\right]}
$$

where the amplitude and phase perturbations $\Delta \bar{X}_{k l}(t), \varphi(t)$ have been introduced. Now, considering that the phase $\varphi(t)$ is a function of the state variables, the following differential equation can be stated:

$$
\begin{aligned}
\dot{\varphi}(t) & =\frac{\partial \varphi}{\partial \bar{x}} \dot{\bar{x}}= \\
& =\sum_{k, l} \bar{G}_{k l}[\bar{X}, \Delta \bar{X}(t)]^{+} \dot{\bar{x}}(t) e^{j\left[k\left(\omega_{0} t+\varphi\right)+l \omega t\right]}= \\
& =\sum_{k, l} Q_{k l}(\bar{X}, \Delta \bar{X}(t), \dot{\varphi}) e^{j\left(k \omega_{0}+l \omega\right) t} e^{j k \varphi(t)}
\end{aligned}
$$

where $\bar{G}_{k l}$ is the vector of the harmonics of $\partial \varphi / \partial \bar{x}$ of order $(k, l)$ and the harmonic components $Q_{k l}$ result from rearranging the product in the second line of (24) in the Fourier basis. In the case $m \omega \simeq n \omega_{0}$, the exponential terms $\pm\left(n \omega_{0}-m \omega\right)$ become dominant in $\varphi$ due to the averaging produced by the time integration. Assuming that the amplitude $A$ is small enough, the amplitude perturbations can be neglected in (24) and the phase perturbation is ruled by the following differential equation :

$$
\begin{aligned}
& \varphi(t) \simeq \int_{0}^{t} B(\dot{\varphi}) \cos \left[n\left(\omega_{0} \tau+\varphi(\tau)\right)-m \omega \tau+\alpha\right] d \tau, \\
& B(\dot{\varphi})=\left|Q_{n,-m}(\bar{X}, \dot{\varphi})\right|
\end{aligned}
$$

Synchronization occurs when:

$$
n\left(\omega_{0}+\dot{\varphi}\right)=m \omega \longrightarrow \varphi=\Delta \omega t+\phi_{0}
$$

with $\Delta \omega=m \omega / n-\omega_{0}$. The synchronized solution is periodic with the fundamental frequency $\omega_{s}=\omega / n=\left(\omega_{0}+\right.$ $\Delta \omega) / m$. If we introduce the expression (26) for $\varphi$ in (25) we obtain:

$$
\frac{\Delta \omega}{B(\Delta \omega)} \simeq \cos \left[n \phi_{0}+\alpha\right]
$$

Now, taking into account that the autonomous frequency $\omega_{a}$ grows monotonically with the parameter $\rho$, an equation of the following form can be obtained:

$$
g(\Delta \rho) \simeq \cos \left[n \phi_{0}+\alpha\right]
$$

If $\Delta \rho$ is considered as an unknown, it is seen that by sweeping $n \phi_{0} \in[0,2 \pi]$ the whole set of $\Delta \rho$ values corresponding to the synchronization range is obtained. The phase $n \phi_{0}$ corresponds to the harmonic $k=n, l=0$ in the expansion (23). Assuming that the amplitude $A$ is small, this harmonic agrees with the $p-t h$ component in the periodic basis $\left\{e^{j k \omega_{s} t}\right\}$, with $p=n m$. Then, when considering the previous approaches, the phase of the $p-t h$ harmonic component of the synchronized solution covers the interval $[0,2 \pi]$ along the synchronization range versus $\Delta \rho$.

Proposition 2: Let $\bar{\Theta}$ be the vector of harmonics of the state variable $\theta(t)$ in the synchronized interval $I(A, \omega, m, n)$. Along this interval, for $A$ small enough, the solutions of system (17) lie in a closed curve in the space $(\bar{\Theta}, \rho)$.

Proof: Let $\left(\bar{\Theta}_{0}, \rho_{0}\right)$ be the non perturbed solution to system (17) for $A=0$. For a small value of the normalized amplitude $A$, this solution becomes $\left(\bar{\Theta}_{0}+\Delta \bar{\Theta}, \rho_{0}+\Delta \rho\right)$, and system (17) can be linearized about the non perturbed solution, obtaining the linear system:

$$
\left.\frac{\partial \bar{H}}{\partial \bar{\Theta}}\right|_{0} \Delta \bar{\Theta}+\left.\frac{\partial \bar{H}}{\partial \rho}\right|_{0} \Delta \rho=A \bar{E}_{n}
$$


System (29) has $2 N+1$ equations and $2 N+2$ unknowns. The system can be balanced by including the additional equation:

$$
\arg \Theta_{p}=\varphi_{p}, \quad p=m n
$$

As it has been demonstrated in Proposition 1, the whole set of synchronized solutions can be obtained by solving system (29)-(30) for all the values of $\varphi_{p} \in[0,2 \pi]$. As the phase $\varphi_{p}$ is varied in the interval $[0,2 \pi]$, the solutions $\bar{\Theta}_{0}+\Delta \bar{\Theta}\left(\varphi_{p}\right)$ and $\rho_{0}+\Delta \rho\left(\varphi_{p}\right)$ of system (29)-(30) trace a closed curve in the space $(\bar{\Theta}, \rho)$

\section{ACKNOWLEDGEMENT}

The authors would like to thank Dr. Franco Ramirez for technical discussions.

\section{REFERENCES}

[1] C. Cosmelli, F. Chiarello, G. D'Agosta, M. Castellano, and G. Torrioli, "Measurements of the decoherence time from energy level quantization in Josephson junctions and rf SQUIDs," IEEE Transactions on Applied Superconductivity, vol. 9, no. 2, pp. 4123-4126, June 1999.

[2] S. K. Dana, D. C. Sengupta, and C. K. Hu, "Spiking and Bursting in Josephson Junction," IEEE Transactions on Circuits and Systems- II: Express Briefs, vol. 53, no. 10, pp. 1031-1034, October 2006.

[3] K. K. Likharev, Dynamics of Josephson Junctions and Circuits. Amsterdam: Gordon and Breach Publishers, 1986.

[4] P. W. Anderson, "Considerations on the flow of superfluid Helium," Progress in Low Temperature Physics, vol. 38, no. 2, pp. 298-310, April 1966.

[5] A. Abidi and L. Chua, "On the dynamics of Josephson-junction circuits," IEE Journal on Electronic Circuits and Systems, vol. 3, no. 4, pp. 186 -200, July 1979.

[6] M. Odyniec and L. O. Chua, "Josephson-junction circuit analysis via integral manifolds," IEEE Trans. on Circuits Syst.-I: Fundamental Theory and Applications, vol. 30, no. 5, May 1983.

[7] — "Josephson-junction circuit analysis via integral manifolds: Part II," IEEE Trans. on Circuits Syst.-I: Fundamental Theory and Applications, vol. 32, no. 1, May 1985.

[8] F. Salam and S. Sastry, "Dynamics of the forced Josephson Junction circuit: The regions of chaos," IEEE Transactions on Circuits and Systems, vol. 32, no. 8, pp. 784-796, Aug 1985.

[9] P. W. Anderson, "The Josephson effect and quantum coherence measurements in superconductors and superfluids," Reviews of Modern Physics, vol. 5, pp. 1-43, 1967.

[10] H. Fack and V. Kose, "Pull-in phenomena of Josephson oscillators," Journal of Applied Physics, vol. 42, pp. 320-322, January 1971.

[11] V. Belykh, N. Pedersen, and O. Soerensen, "Shunted-Josephson-Junction model. II-The nonautonomous case," Physical Review B, vol. 16, no. 11, pp. 4860-4871, 1977.

[12] S. Sastry, Nonlinear Systems. Analysis, Stability and Control. SpringerVerlag, 1999.

[13] E. J. Doedel et al., AUTO 2000: Continuation and bifurcation software for ordinary differential equations, 2002.

[14] H. B. Keller, Lectures on Numerical Methods In Bifurcation Problems. Tata Institute Of Fundamental Research, January 1986.

[15] T. J. Aprille and T. N. Trick, "Steady-state analysis of nonlinear circuits with periodic inputs," Proceedings of the IEEE, vol. 60, no. 1, pp. 108114, January 1972.

[16] F. Schilder, H. M. Osinga, and W. Vogt, "Continuation of quasi-periodic invariant tori," SIAM Journal on Applied Dynamical Systems, vol. 4(3), pp. 459-488, January 2005.

[17] F. Schilder, W. Vogt, S. Schreiber, and H. M. Osinga, "Fourier methods for quasi-periodic oscillations," International Journal for Numerical Methods in Engineering, vol. 67(5), pp. 629-671, January 2006.

[18] K. S. Kundert, J. K. White, and A. Sangiovanni-Vincentelli, Steady-State Methods for Simulating Analog and Microwave Circuits. Norwell, Ma.: Kluwer Academic Publishers, 1990.

[19] C. K. Bak and N. Pedersen, "Josephson junction analog and quasiparticle-pair current," Applied Physics Letters, vol. 22, no. 4, pp. 149-150, February 1973
[20] S. Sancho, A. Suarez, and J. Chuan, "General envelope-transient formulation of phase-locked loops using three time scales," IEEE Transactions on Microwave Theory and Techniques, vol. 52, no. 4, pp. 1310-1320, 2004.

[21] J. Dominguez, S. Sancho, and A. Suarez, "Time-frequency formulation for the nonlinear analysis of coupled phase-locked loops," IEEE Transactions on Microwave Theory and Techniques, vol. 56, no. 12, pp. 2838-2850, Dec. 2008.

[22] H. R. Mohebbi and A. H. Majedi, "Analysis of series-connected discrete Josephson transmission line," IEEE Transactions on Microwave Theory and Techniques, vol. 57, no. 8, pp. 1865-1873, August 2009.

[23] H. Salehi, R. R. Mansour, and A. H. Majedi, "Nonlinear Josephson lefthanded transmission lines," IET Microwaves, Antennas \& Propagation, vol. 1, no. 1, pp. 69-72, August 2007.

[24] R. J. Schoelkopf, J. Zmuidzinas, T. G. Phillips, H. G. LeDuc, and J. A. Stern, "Measurements of noise in Josephson-effect mixers," IEEE Transactions on Microwave Theory and Techniques, vol. 43, no. 4, pp. 977-983, April 1995.

[25] P. A. Rosenthal and E. N. Grossman, "Terahertz Shapiro Steps in high temperature SNS Josephson Junctions," IEEE Transactions on Microwave Theory and Techniques, vol. 42, no. 4, pp. 707-714, April 1994.

[26] P. Russer and J. A. Russer, "Nanoelectronic RF Josephson devices," IEEE Transactions on Microwave Theory and Techniques, vol. 59, no. 10 , pp. 2685-2701, October 2011.

[27] Guide to Harmonic Balance Simulation in ADS, Agilent Technologies. Palo Alto, CA, U.S.A., 2004.

[28] V. Rizzoli and A. Neri, "State of the art and present trends in nonlinear microwave cad techniques," IEEE Transactions on Microwave Theory and Techniques, vol. 36, no. 2, pp. 343-356, February, 1988.

[29] J. K. Hale and S. M. V. Lunel, Introduction to Functional Differential Equations. New York: Springer-Verlag, 1993.

[30] L. Chua and A. Ushida, "A switching-parameter algoritm for finding multiple solutions on nonlinear resistive circuits," Int. J. Circuit Theory Appl., vol. 4, no. 3, May 1976.

[31] F. L. Traversa, F. Bonani, and S. Donati Guerrieri, "A frequency-domain approach to the analysis of stability and bifurcations in nonlinear systems described by differential-algebraic equations," Int. J. Circuit Theory \& App, vol. 36, no. 4, pp. 421-439, June 2008

[32] F. Bonani and M. Gilli, "Analysis of stability and bifurcations of limit cycles in Chua's Circuit through the Harmonic-Balance approach," IEEE Transactions on Circuits and Systems-I: Fundamental Theory and Applications, vol. 46, no. 8, pp. 881-890, August 1999.

[33] S. Sancho, A. Suarez, and P. San Roman, "Floquet Analysis of the Intermittence Route to Chaos through a Pitchfork bifurcation," IEEE Transactions on Circuits and Systems, Fundamental Theory And Applications, vol. 48, no. 3, pp. 374-377, March 2001.

[34] J. M. T. Thompson and H. B. Stewart, Nonlinear Dynamics and Chaos. John Wiley \& Sons, 2002.

[35] A. Suarez, Analysis and Design of Autonomous Microwave Circuits. Wiley, 2009.

[36] I. Schmideg, "Harmonic synchronization of nonlinear oscillators," Proceedings of the IEEE, vol. 59, no. 8, pp. 1250-1251, August 1971.

[37] A. Buonomo and A. Lo Schiavo, "Analytical approach to the study of injection-locked frequency dividers," IEEE Transactions on Circuits and Systems-I, vol. 60, no. 1, pp. 51-62, 2013.

[38] I. Ali, A. Banerjee, A. Mukherjee, and B. N. Biswas, "Study of injection locking with amplitude perturbation and its effect on pulling of oscillator," IEEE Transactions on Circuits and Systems-I, vol. 59, no. 1 pp. 137-147, 2012.

[39] A. Amann, M. P. Mortell, E. P. O'Reilly, M. Quinlan, and D. Rachinskii, "Mechanism of synchronization in frequency dividers," IEEE Transactions on Circuits and Systems-I, vol. 56, no. 1, pp. 190199, 2009.

[40] A. Buonomo and A. Lo Schiavo, "Nonlinear dynamics of divide-by-two frequency dividers in locked mode," International Journal of Circuit Theory and Applications, no. DOI:10.1002/cta.1888.

[41] S. Wiggins, Introduction to Applied Nonlinear Dynamical Systems and Chaos. New York: Springer-Verlag, 1990.

[42] T. Matsumoto, "Chaos in Electronic Circuits," Proceedings of the IEEE vol. 75, no. 8, pp. 1033-1057, August 1987.

Sergio Sancho was born in Santurce, Spain. In 1997 received the degree in Physics from Basque Country University. In 1998 he joined the Communications Engineering Department of the University of Cantabria, 
Spain, where he received the Ph.D. degree in Electronic Engineering in February 2002. At present, he works at the University of Cantabria, as an Associate Professor of its Communications Engineering Department. His research interests include the nonlinear analysis of microwave circuits and frequency synthesizers, the investigation of chaotic regimes and the phasenoise analysis.

Almudena Suarez (F'12) was born in Santander, Spain. She received the degree in electronic physics and the Ph.D. degree from the University of Cantabria, Santander, Spain, in 1987 and 1992, respectively, and the Ph.D. degree in electronics from the University of Limoges, Limoges, France, in 1993. At present, she is a Full Professor at the University of Cantabria, and a member of its Communications Engineering Department. She has authored the book Analysis and design of autonomous microwave circuits for the publisher IEEE-Wiley and co-authored the book Stability analysis of microwave circuits for the publisher Artech-House. She belongs to the technical committees of the IEEE International Microwave Symposium and European Microwave Conference. She was an IEEE Distinguished Microwave Lecturer for the period 2006-2008. She is the coordinator of the research area "Electronic and Communications Technology" of the Spanish national evaluation agency ANEP. 